\title{
Parameter Space of Shock Formation in Adiabatic Flows ${ }^{1}$
}

\author{
Ju-Fu Lu, ${ }^{2,3}$ K.N. Yu, ${ }^{3}$ F. Yuan, ${ }^{2}$ and E. C. M. Young ${ }^{3}$ \\ ${ }^{2}$ Center for Astrophysics, University of Science and Technology of \\ China, Hefei, Anhui, 230026, China \\ ${ }^{3}$ Department of Physics and Materials Science, City University of Hong \\ Kong, Tat Chee Avenue, Kowloon, Hong Kong
}

We study shock formation in a stationary, axisymmetric, adiabatic flow of a perfect fluid in the equatorial plane of a Kerr geometry. For such a flow, there exist two intrinsic constants of motion along a fluid world line, namely the specific total energy, $E=-h u_{t}$, and the specific angular momentum. $l=-u_{\varphi} / u_{t}$, where the $u_{\mu}$ 's are the four velocity components, $h$ is the specific enthalpy, i.e., $h=(P+\varepsilon) / \rho$, with $P, \varepsilon$, and $\rho$ being the pressure, the mass-energy density. and the rest-mass density, respectively.

As shown in Fig. 1 (Fig. 1 a is for a Schwarzschild black hole, i.e. the hole's specific angular momentum $a=0$; Fig. $1 \mathrm{~b}$ is for a rapid Kerr hole, i.e. $a=0.99 M$, where $M$ is the black-hole mass, and prograde flows: and Fig. 1c is for $a=0.99 \mathrm{M}$ and retrograde flows), in the parameter space spanned by $E$ and $l$ there is a strictly defined region bounded by four lines: three characteristic functional curves $l_{k}(E), l_{\max }(E)$, and $l_{\min }(E)$, and the vertical line $E=1$. Only such a flow with parameters located within this region can have two physically realizable sonic points, the inner one $r_{i n}$, and the outer one $r_{\text {out }}$. In between there is still one more, but unrealizable, sonic point, $r_{\text {mid }}$. The region is divided by another characteristic functional curve $l_{c}(E)$ into two parts: in region I $(=$ $\mathrm{Ia}+\mathrm{Ib}$ ) only $r_{\text {out }}$ is realized in a shock-free global solution (i.e., that joining the black-hole horizon to large distances), while in region II ( $=\mathrm{Ila}+\mathrm{IIb})$ only $r_{\text {in }}$ is realized.

There further exists a new strictly defined function, $l_{s}(E)$, drawn in Fig. 1 as two curves $l_{s \mathrm{I}}$ and $l_{s \mathrm{I}}$, for an accretion flow with parameters $E$ and $l$ located within region Ia (bounded by lines $l_{c}, l_{s I}$, and $E=1$ ), or a wind flow with $E$ and $l$ belonging to region IIa (bounded by $l_{k}, l_{s I 1}, l_{c}$, and $E=1$ ), there are four formal shock locations, denoted by $r_{s 1}, r_{s 2}, r_{s 3}$, and $r_{s 4}$, which are related to the three formal locations of the sonic point as $r_{s 1}<r_{i n}<r_{s 2}<r_{\text {mid }}<r_{s 3}<$ $r_{\text {out }}<r_{s 4}$; while for an accretion flow belonging to region Ib (bounded by $l_{s \mathrm{I}}$, $l_{\min }$, and $E=1$ in Figs. $1 \mathrm{a}$ and $1 \mathrm{~b}$, and by $l_{s \mathrm{I}}, l_{\max }$, and $E=1$ in Fig. 1c), or a wind flow belonging to region IIb (bounded by $l_{k}, l_{\max }$, and $l_{s I I}$ in Figs. $1 \mathrm{a}$ and $1 \mathrm{~b}$, and by $l_{k}, l_{\min }$, and $l_{s I I}$ in Fig. 1c), there are only two formal shock locations, denoted by $r_{s 1}$ and $r_{s 4}$, satisfying $r_{s 1}<r_{i n}, r_{o u t}<r_{s 4}$. It is clear that for an accretion flow onto a black hole, both $r_{s 1}$ and $r_{s 4}$ can be ruled out by employing boundary conditions that the flow must be supersonic when crossing the black-hole horizon and subsonic when starting at a large distance (however,

${ }^{1}$ This work is supported by the National Natural Science Foundation of China. 


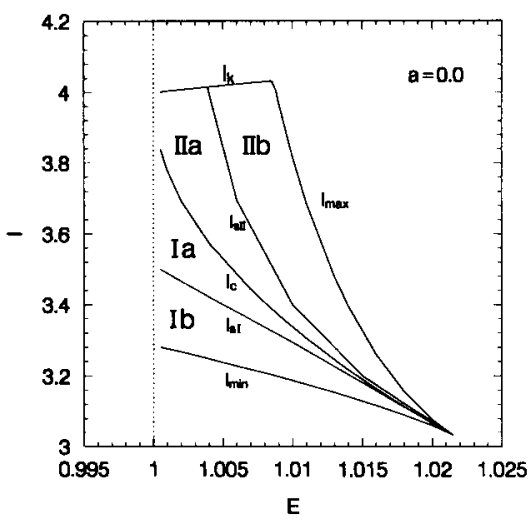

Fig.1a

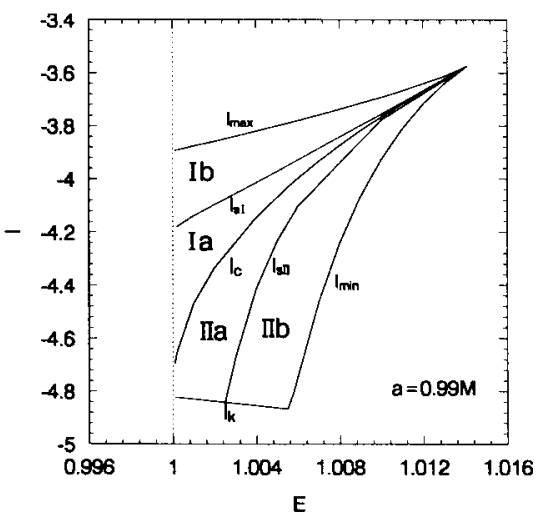

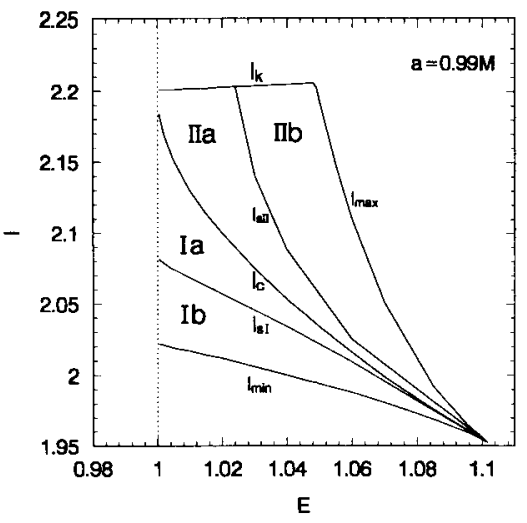

Fig.1b

Fig.1c

for accretion onto a neutron star, $r_{s 1}$ is a possible shock location); while for a wind flow $r_{s 1}$ is not possible either, but $r_{s 4}$ is possible if the flow is terminally subsonic. The possibilities of shocks at $r_{s 2}$ and $r_{s 3}$ cannot be judged in this way, we have therefore made stability analysis and have been able to reach the following conclusion: for accretion flows the shock at $r_{s .3}$ is always stable, while the shock at $r_{s 2}$ is unstable except when the value of $l$ is very close to that of $l_{s}$; for wind flows the shock at $r_{s 3}$ is always unstable, while the shock at $r_{s 2}$, is stable except when $l$ is very close to $l_{s}$. 